\title{
A few remarks on the operator norm of random Toeplitz matrices
}

\author{
Radosław Adamczak \\ University of Warsaw *†
}

November 8, 2018

\begin{abstract}
We present some results concerning the almost sure behaviour of the operator norm or random Toeplitz matrices, including the law of large numbers for the norm, normalized by its expectation (in the i.i.d. case). As tools we present some concentration inequalities for suprema of empirical processes, which are refinements of recent results by Einmahl and Li.
\end{abstract}

Keywords: random Toeplitz matrices

AMS classification: 15A52, 60F15

\section{Introduction}

For a sequence of independent real random variables $X_{0}, X_{1}, \ldots$, consider the associated sequence of random Toeplitz matrices, given by

$$
T_{n}=\left[\begin{array}{cccccc}
X_{0} & X_{1} & X_{2} & \ldots & X_{n-2} & X_{n-1} \\
X_{1} & X_{0} & X_{1} & & & X_{n-2} \\
X_{2} & X_{1} & X_{0} & & & \vdots \\
\vdots & & & \ddots & & \vdots \\
X_{n-2} & & & & X_{0} & X_{1} \\
X_{n-1} & X_{n-2} & \ldots & \ldots & X_{1} & X_{0}
\end{array}\right]=\left[X_{|i-j|}\right]_{1 \leq i, j \leq n} .
$$

In two recent articles, Bryc, Dembo and Jiang $[5]$ and independently Hammond and Miller [9] addressed the questions raised previously in Bai's paper [3], concerning the asymptotic behaviour of the spectral measure of random

*University of Warsaw, Institute of Mathematics, ul. Banacha 2, 02-097 Warszawa, Poland, e-mail: radamcz@mimuw.edu.pl.

†This work was completed during the author's stay at the University of Alberta, Edmonton, Canada within the PIMS postdoctoral fellowship. 
Toeplitz matrices generated by i.i.d. mean zero, variance one random variables. They showed that the spectral measure of $n^{-1 / 2} T_{n}$ with probability one converges weakly to a nonrandom measure, independent of the distribution of the underlying i.i.d. sequence. Their results were later complemented by Bose and Sen [4] and Meckes [12] who analyzed the asymptotic behaviour of the spectral norm of a random Toeplitz matrix. Bose and Sen proved a law of large numbers in the case of i.i.d. random variables with positive mean and finite variance. Meckes showed that if all $X_{i}$ 's are centered and uniformly subgaussian then $\mathbb{E}\left\|T_{n}\right\| \simeq \sqrt{n \log n}$ and the same holds with probability one for $\left\|T_{n}\right\|$, provided that $X_{i}$ 's have some concentration of measure property.

In the present article we are interested in extending the results of 12 to i.i.d. mean zero random variables with finite variance as well as to independent non-identically distributed variables, which satisfy some moment bounds. In particular, we prove that if $X_{0}, X_{1}, X_{2}, \ldots$ are i.i.d. random variables, $\mathbb{E} X_{0}=0$ and $0<\mathbb{E} X_{0}^{2}<\infty$, then

$$
\lim _{n \rightarrow \infty} \frac{\left\|T_{n}\right\|}{\mathbb{E}\left\|T_{n}\right\|}=1 \text { a.s. }
$$

This is the statement of Theorem 5 in Section 3.2 .2

The above result, together with some additional arguments, may be used to prove that if $X_{0}, X_{1}, X_{2}, \ldots$ are i.i.d. random variables, then

$$
\limsup _{n \rightarrow \infty} \frac{\left\|T_{n}\right\|}{\sqrt{n \log n}}<\infty \text { a.s. }
$$

if and only if $\mathbb{E} X_{0}=0$ and $\mathbb{E} X_{0}^{2}<\infty$, which is the content of Corollary 3 , Section 3.2.2.

In the framework of independent, but not necessarily identically distributed variables, we give sufficient conditions for (1) to hold (Proposition 1 Section 3.2.1)

Our tools come from the classical probability in Banach spaces theory and are based on concentration inequalities for sums of independent Banach space valued variables. In particular, the proofs are inspired by a recent paper by Einmahl and $\mathrm{Li}[\underline{6}$, in which a very general law of the iterated logarithm for sums of i.i.d. variables in Banach spaces has been characterized. The other crucial ingredient is the original argument by Meckes which is applied conditionally.

The organization of the paper is as follows. In the next section we present the basic concentration results for sums of independent random variables. Next, in Section 3, we pass to the original problem, concerning random matrices. First we obtain some useful estimates for the expected operator norm and then we prove results on the almost sure behaviour. We conclude with two exponential inequalities. 
In the article we will deal both with absolute constants and constants depending on some parameters, which will be explicitly stated or clear from the context. In both cases the values of constants may change from line to line.

Acknowledgements The author would like to thank Prof. Mark Meckes and Prof. Włodzimierz Bryc for introducing him to the problem and valuable remarks concerning the early version of the article.

\section{Concentration of measure for suprema of em- pirical processes}

In this section we introduce the basic tool required for the analysis of the almost sure behaviour of $\left\|T_{n}\right\|$, i.e. concentration inequalities for sums of independent Banach space valued random variables. The following exposition is motivated by the work of Einmahl and Li. In particular, our Theorem 2 is a version of Theorem 3.1. from [6].

All the concentration results presented below are based on the celebrated inequality by Talagrand [13. We will use a recent version by Klein and Rio, which gives the optimal constant 2 in front of the variance. We would also like to mention that since the natural normalization of the operator norm of Toeplitz matrices is $\sqrt{n \log n}$, which is much bigger than the classical law of the iterated logarithm normalization, this optimal value of the constant is not important for our applications (in fact any other constant would work). However, since inequalities for sums of Banach space valued variables with optimal value of this constant are of independent interest and obtaining them does not require much additional work, we present the results of this section in a slightly larger generality, than necessary for subsequent applications.

Let us first recall the result due to Klein and Rio

Theorem 1 ([11], Theorems 1.1, 1.2). Let $X_{1}, X_{2}, \ldots, X_{n}$ be independent random variables with values in a measurable space $(S, \mathcal{S})$ and let $\mathcal{F}$ be a countable class of measurable functions $f: \mathcal{S} \rightarrow[-M, M]$, such that for all $i, \mathbb{E} f\left(X_{i}\right)=0$. Consider the random variable

$$
Z=\sup _{f} \sum_{i=1}^{n} f\left(X_{i}\right)
$$

Then, for all $t \geq 0$,

$$
\mathbb{P}(Z \geq \mathbb{E} Z+t) \leq \exp \left[-\frac{t^{2}}{2\left(\sigma^{2}+2 M \mathbb{E} Z\right)+3 M t}\right]
$$

and

$$
\mathbb{P}(Z \leq \mathbb{E} Z-t) \leq \exp \left[-\frac{t^{2}}{2\left(\sigma^{2}+2 M \mathbb{E} Z\right)+3 M t}\right]
$$


where

$$
\sigma^{2}=\sup _{f \in \mathcal{F}} \sum_{i=1}^{n} \mathbb{E} f\left(X_{i}\right)^{2} .
$$

The following corollary was derived from the above theorem by Einmahl and Li [6] (they stated only the first part, concerning the upper tail but their proof yields also the other inequality).

Corollary 1. Let $X_{1}, \ldots, X_{n}$ be independent mean zero random variables with values in a separable Banach space B. Assume that $\left\|X_{i}\right\|_{\infty} \leq M$ for every $i$ and let

$$
\sigma^{2}=\sup _{f \in B^{*},\|f\| \leq 1} \sum_{i} \mathbb{E} f\left(X_{i}\right)^{2} .
$$

Consider the random variable

$$
Z=\left\|\sum_{i=1}^{n} X_{i}\right\|
$$

Then for all $0<\eta \leq 1, \delta>0$ there exists a constant $K=K(\eta, \delta)$, such that for all $t \geq 0$,

$$
\begin{aligned}
\mathbb{P}\left(\max _{1 \leq k \leq n}\left\|\sum_{i=1}^{k} X_{i}\right\| \geq(1+\eta) \mathbb{E} Z+t\right) \leq & \exp \left[-\frac{t^{2}}{2(1+\delta) \sigma^{2}}\right] \\
& +\exp \left[-\frac{t}{K \max _{i}\left\|X_{i}\right\|_{\infty}}\right]
\end{aligned}
$$

and

$$
\mathbb{P}(Z \leq(1-\eta) \mathbb{E} Z-t) \leq \exp \left[-\frac{t^{2}}{2(1+\delta) \sigma^{2}}\right]+\exp \left[-\frac{t}{K \max _{i}\left\|X_{i}\right\|_{\infty}}\right] .
$$

Using the above result, Einmahl and Li [6] obtained an infinite dimensional version of the Fuk-Nagaev inequality for the deviation of maxima of partial sums of independent random variables [7]. For our purposes we will need a slightly refined version of their estimate, given in the following

Theorem 2. In the setting of Corollary 1 assume that for some $p \geq 1$ and all $i, \mathbb{E}\left\|X_{i}\right\|^{p}<\infty$. Then for all $0<\eta \leq 1, \delta>0$ there exists a constant $C=C(p, \eta, \delta)$, such that for all $t \geq 0$,

$$
\begin{aligned}
& \mathbb{P}\left(\max _{1 \leq k \leq n}\left\|\sum_{i=1}^{k} X_{i}\right\| \geq(1+\eta) \mathbb{E} Z+t\right) \\
& \quad \leq \exp \left[-\frac{t^{2}}{2(1+\delta) \sigma^{2}}\right]+C \mathbb{E} \max _{1 \leq i \leq n}\left\|X_{i}\right\|^{p} / t^{p}
\end{aligned}
$$

and

$$
\begin{aligned}
& \mathbb{P}(Z \leq(1-\eta) \mathbb{E} Z-t) \\
& \quad \leq \exp \left[-\frac{t^{2}}{2(1+\delta) \sigma^{2}}\right]+C \mathbb{E} \max _{1 \leq i \leq n}\left\|X_{i}\right\|^{p} / t^{p}
\end{aligned}
$$


The proof we give below follows quite closely the original argument of Einmahl and $\mathrm{Li}$ and is based on the Hoffmann-Jørgensen inequality. The difference is that we use it in the moment version, which in our opinion simplifies the argument and also allows to replace $\sum_{i} \mathbb{E}\left\|X_{i}\right\|^{p}$, appearing in the original estimate by Einmahl and Li, by $\mathbb{E} \max _{i}\left\|X_{i}\right\|^{p}$ (this will be useful when dealing with Toeplitz matrices generated by non i.i.d. variables). A somewhat similar application to moment estimates appeared in [8], whereas [2] contains an analogous derivation in the context of exponential inequalities for unbounded empirical processes (its final version was also influenced by results of Einmahl and $\mathrm{Li}$ ). We state it at the end of the present section.

Proof. For $\varepsilon=\varepsilon(\delta)>0$ (whose value will be determined later) consider truncated variables $Y_{i}=X_{i} \mathbf{1}_{\left\{\left\|X_{i}\right\| \leq \rho\right\}}$. Set also $R_{i}=X_{i}-Y_{i}$.

We have

$$
\max _{1 \leq k \leq n}\left\|\sum_{i=1}^{k} X_{i}\right\| \leq \max _{1 \leq k \leq n}\left\|\sum_{i=1}^{k}\left(Y_{i}-\mathbb{E} Y_{i}\right)\right\|+\max _{1 \leq k \leq n}\left\|\sum_{i=1}^{k}\left(R_{i}-\mathbb{E} R_{i}\right)\right\|
$$

and

$$
\left\|\sum_{i=1}^{n} X_{i}\right\| \geq\left\|\sum_{i=1}^{n}\left(Y_{i}-\mathbb{E} Y_{i}\right)\right\|-\left\|\sum_{i=1}^{n}\left(R_{i}-\mathbb{E} R_{i}\right)\right\|,
$$

where we used the fact that $\mathbb{E} Y_{i}+\mathbb{E} R_{i}=0$.

Similarly, using Jensen's inequality, we obtain

$$
\mathbb{E}\left\|\sum_{i=1}^{n}\left(Y_{i}-\mathbb{E} Y_{i}\right)\right\|-2 \mathbb{E}\left\|\sum_{i=1}^{n} R_{i}\right\| \leq \mathbb{E} Z \leq \mathbb{E}\left\|\sum_{i=1}^{n}\left(Y_{i}-\mathbb{E} Y_{i}\right)\right\|+2 \mathbb{E}\left\|\sum_{i=1}^{n} R_{i}\right\| .
$$

Thus, setting $A=\mathbb{E} \max _{1 \leq k \leq n}\left\|\sum_{i=1}^{k} R_{i}\right\|$, we get

$$
\begin{aligned}
& \mathbb{P}\left(\max _{1 \leq k \leq n}\left\|\sum_{i=1}^{k} X_{i}\right\| \geq(1+\eta) \mathbb{E} Z+t\right) \\
& \leq \mathbb{P}\left(\max _{1 \leq k \leq n}\left\|\sum_{i=1}^{k}\left(Y_{i}-\mathbb{E} Y_{i}\right)\right\| \geq(1+\eta) \mathbb{E} Z+(1-\varepsilon) t\right) \\
& \quad+\mathbb{P}\left(\max _{1 \leq k \leq n}\left\|\sum_{i=1}^{k}\left(R_{i}-\mathbb{E} R_{i}\right)\right\| \geq \varepsilon t\right) \\
& \leq \mathbb{P}\left(\max _{1 \leq k \leq n}\left\|\sum_{i=1}^{k}\left(Y_{i}-\mathbb{E} Y_{i}\right)\right\| \geq(1+\eta) \mathbb{E}\left\|\sum_{i=1}^{k}\left(Y_{i}-\mathbb{E} Y_{i}\right)\right\|-4 A+(1-\varepsilon) t\right) \\
& \quad+\mathbb{P}\left(\max _{1 \leq k \leq n}\left\|\sum_{i=1}^{k}\left(R_{i}-\mathbb{E} R_{i}\right)\right\| \geq \varepsilon t\right)
\end{aligned}
$$


and similarly

$$
\begin{aligned}
& \mathbb{P}(Z \leq(1-\eta) \mathbb{E} Z-t) \\
& \leq \mathbb{P}\left(\left\|\sum_{i=1}^{n}\left(Y_{i}-\mathbb{E} Y_{i}\right)\right\| \leq(1-\eta) \mathbb{E} Z-(1-\varepsilon) t\right) \\
& \quad+\mathbb{P}\left(\left\|\sum_{i=1}^{n}\left(R_{i}-\mathbb{E} R_{i}\right)\right\| \geq \varepsilon t\right) \\
& \leq \mathbb{P}\left(\left\|\sum_{i=1}^{n}\left(Y_{i}-\mathbb{E} Y_{i}\right)\right\| \leq(1-\eta) \mathbb{E}\left\|\sum_{i=1}^{n}\left(Y_{i}-\mathbb{E} Y_{i}\right)\right\|-(1-\varepsilon) t+2 A\right) \\
& \quad+\mathbb{P}\left(\left\|\sum_{i=1}^{n}\left(R_{i}-\mathbb{E} R_{i}\right)\right\| \geq \varepsilon t\right) .
\end{aligned}
$$

We would like to apply Corollary 1 to bound first summands on right hand sides of the above inequalities and the Markov inequality to bound the deviation of the remainder. Thus the aim is now to find a good truncation level $\rho$, which would ensure that $A \simeq \varepsilon t$ and both

$$
e^{-t(1-\mathcal{O}(\varepsilon)) / K \max _{i}\left\|Y_{i}-\mathbb{E} Y_{i}\right\|_{\infty}}, \mathbb{P}\left(\max _{1 \leq k \leq n}\left\|\sum_{i=1}^{k}\left(R_{i}-\mathbb{E} R_{i}\right)\right\| \geq \varepsilon t\right)
$$

are of order $t^{-p} \mathbb{E} \max _{i \leq n}\left\|X_{i}\right\|^{p}$, where $K=K(\eta, \delta)$ is the constant from Corollary 1.

Recall now the classical Hoffmann-Jørgensen inequality (e.g. [10, Chapter 6, Proposition 6.8., inequality (6.8)), which asserts that

$$
\mathbb{E} \max _{1 \leq k \leq n}\left\|\sum_{i=1}^{k} R_{i}\right\|^{p} \leq 2 \cdot 4^{p} \mathbb{E} \max _{1 \leq i \leq n}\left\|R_{i}\right\|^{p}+2\left(4 t_{0}\right)^{p},
$$

where

$$
t_{0}=\inf \left\{t>0: \mathbb{P}\left(\max _{1 \leq k \leq n}\left\|\sum_{i=1}^{k} R_{i}\right\|>t\right) \leq\left(2 \cdot 4^{p}\right)^{-1}\right\} .
$$

The idea (following [8]) is to truncate at a level which would allow to take $t_{0}=0$. Let us thus set

$$
\rho^{p}=2 \cdot 4^{p} \cdot \mathbb{E} \max _{1 \leq i \leq n}\left\|X_{i}\right\|^{p} .
$$

Then $\mathbb{P}\left(\max _{1 \leq k \leq n}\left\|\sum_{i=1}^{k} R_{i}\right\|>0\right) \leq \mathbb{P}\left(\max _{1 \leq i \leq n}\left\|X_{i}\right\|>\rho\right) \leq\left(2 \cdot 4^{p}\right)^{-1}$ by the definition of $R_{i}$ and the Markov inequality. Thus

$$
\mathbb{E} \max _{1 \leq k \leq n}\left\|\sum_{i=1}^{k} R_{i}\right\|^{p} \leq 2 \cdot 4^{p} \cdot \mathbb{E} \max _{1 \leq i \leq n}\left\|X_{i}\right\|^{p}
$$


and also

$$
\mathbb{E} \max _{1 \leq k \leq n}\left\|\sum_{i=1}^{k} R_{i}\right\| \leq 2^{1 / p} \cdot 4 \cdot\left(\mathbb{E} \max _{1 \leq i \leq n}\left\|X_{i}\right\|^{p}\right)^{1 / p} .
$$

Notice, that we can assume that $\mathbb{E} \max _{1 \leq i \leq n}\left\|X_{i}\right\|^{p} \leq \varepsilon^{p} t^{p}$, since otherwise the statement of the corollary becomes trivial ( $\varepsilon$ is a function of $\delta$, so it can be used to define $C$ such that right hand sides of the inequalities in question are greater than 1 , if $\left.\mathbb{E} \max _{1 \leq i \leq n}\left\|X_{i}\right\|^{p}>\varepsilon^{p} t^{p}\right)$.

Therefore we have

$$
A=\mathbb{E} \max _{1 \leq k \leq n}\left\|\sum_{i=1}^{k} R_{i}\right\| \leq 8 t \varepsilon .
$$

Also, by Jensen's inequality

$$
\mathbb{E} \max _{1 \leq k \leq n}\left\|\sum_{i=1}^{k}\left(R_{i}-\mathbb{E} R_{i}\right)\right\|^{p} \leq 2^{p} \mathbb{E} \max _{1 \leq k \leq n}\left\|\sum_{i=1}^{k} R_{i}\right\|^{p} \leq 2 \cdot 8^{p} \mathbb{E} \max _{1 \leq i \leq n}\left\|X_{i}\right\|^{p},
$$

so Markov's inequality gives

$$
\mathbb{P}\left(\max _{1 \leq k \leq n}\left\|\sum_{i=1}^{k}\left(R_{i}-\mathbb{E} R_{i}\right)\right\| \geq \varepsilon t\right) \leq 2 \cdot 8^{p} \varepsilon^{-p} \frac{\mathbb{E} \max _{1 \leq i \leq n}\left\|X_{i}\right\|^{p}}{t^{p}}
$$

Combining the above estimates with inequalities (4), (5) and (7) gives

$$
\begin{aligned}
& \mathbb{P}\left(\max _{1 \leq k \leq n}\left\|\sum_{i=1}^{k} X_{i}\right\| \geq(1+\eta) \mathbb{E} Z+t\right) \\
& \leq \mathbb{P}\left(\max _{1 \leq k \leq n}\left\|\sum_{i=1}^{k}\left(Y_{i}-\mathbb{E} Y_{i}\right)\right\| \geq(1+\eta) \mathbb{E}\left\|\sum_{i=1}^{k}\left(Y_{i}-\mathbb{E} Y_{i}\right)\right\|+(1-33 \varepsilon) t\right) \\
& \quad+2 \cdot 8^{p} \varepsilon^{-p} \frac{\mathbb{E} \max _{1 \leq i \leq n}\left\|X_{i}\right\|^{p}}{t^{p}}
\end{aligned}
$$

and

$$
\begin{aligned}
& \mathbb{P}(Z \leq(1-\eta) \mathbb{E} Z-t) \\
& \leq \mathbb{P}\left(\left\|\sum_{i=1}^{n}\left(Y_{i}-\mathbb{E} Y_{i}\right)\right\| \leq(1-\eta) \mathbb{E}\left\|\sum_{i=1}^{n}\left(Y_{i}-\mathbb{E} Y_{i}\right)\right\|-(1-17 \varepsilon) t\right) \\
& \quad+2 \cdot 8^{p} \varepsilon^{-p} \frac{\mathbb{E} \max _{1 \leq i \leq n}\left\|X_{i}\right\|^{p}}{t^{p}} .
\end{aligned}
$$

Let us notice that for every $f$ in the unit ball of $B^{*}$ we have $\mathbb{E}\left[f\left(Y_{i}-\mathbb{E} Y_{i}\right)\right]^{2}=$ $\mathbb{E}\left[f\left(Y_{i}\right)-\mathbb{E} f\left(Y_{i}\right)\right]^{2} \leq \mathbb{E} f\left(Y_{i}\right)^{2} \leq \mathbb{E} f\left(X_{i}\right)^{2}$. Thus, by Corollary 1 we can write 


$$
\begin{aligned}
& \mathbb{P}\left(\max _{1 \leq k \leq n}\left\|\sum_{i=1}^{k} X_{i}\right\| \geq(1+\eta) \mathbb{E} Z+t\right), \quad \mathbb{P}(Z \leq(1-\eta) \mathbb{E} Z-t) \\
& \leq \exp \left[-\frac{t^{2}(1-33 \varepsilon)^{2}}{2(1+\delta) \sigma^{2}}\right]+\exp \left[-\frac{(1-33 \varepsilon) t}{2 K(\eta, \delta) \rho}\right]+2 \cdot 8^{p} \varepsilon^{-p} \frac{\mathbb{E} \max _{1 \leq i \leq n}\left\|X_{i}\right\|^{p}}{t^{p}} .
\end{aligned}
$$

Since $\exp (-x) \leq C_{p} x^{-p}$, we have for $\varepsilon<1 / 66$,

$$
\begin{aligned}
\exp \left[-\frac{(1-33 \varepsilon) t}{2 K(\eta, \delta) \rho}\right] & \leq C_{p}[K(\eta, \delta) \rho]^{p} t^{-p}=C_{p} K(\eta, \delta)^{p} \cdot 2 \cdot 4^{p} \frac{\mathbb{E} \max _{1 \leq i \leq n}\left\|X_{i}\right\|^{p}}{t^{p}} \\
& \leq C(p, \eta, \delta) \frac{\mathbb{E} \max _{1 \leq i \leq n}\left\|X_{i}\right\|^{p}}{t^{p}} .
\end{aligned}
$$

To finish the proof it is enough to apply the last two inequalities with $\delta / 2$ instead of $\delta$ and $\varepsilon$ small enough to ensure that

$$
(1+\delta / 2)(1-33 \varepsilon)^{-2} \leq(1+\delta) .
$$

As announced, we would also like to state a similar result, obtained in [2], valid for variables satisfying stronger, exponential integrability conditions. We

will use it to obtain concentration inequalities for $\left\|T_{n}\right\|$ (see Proposition 2 in Section 3.3 below).

Theorem 3. In the setting of Corollary 1, assume that for some $0<\alpha \leq 1$ and all $i,\left\|X_{i}\right\|_{\psi_{\alpha}}<\infty$. For all $0<\eta<1$ and $\delta>0$, there exists a constant $C=C(\alpha, \eta, \delta)$, such that for all $t \geq 0$,

$$
\begin{aligned}
& \mathbb{P}(Z \geq(1+\eta) \mathbb{E} Z+t) \\
& \leq \exp \left[-\frac{t^{2}}{2(1+\delta) \sigma^{2}}\right]+3 \exp \left[-\left(\frac{t}{C\left\|\max _{i}\right\| X_{i}\|\|_{\psi_{\alpha}}}\right)^{\alpha}\right]
\end{aligned}
$$

and

$$
\begin{aligned}
& \mathbb{P}(Z \leq(1-\eta) \mathbb{E} Z-t) \\
& \leq \exp \left[-\frac{t^{2}}{2(1+\delta) \sigma^{2}}\right]+3 \exp \left[-\left(\frac{t}{C\left\|\max _{i}\right\| X_{i}\|\|_{\psi_{\alpha}}}\right)^{\alpha}\right]
\end{aligned}
$$

\section{Random Toeplitz matrices}

In this part we will focus on the initial problem and prove several results, concerning the operator norm of random Toeplitz matrices. The consecutive sections will be devoted to properties of the expectation, almost sure behaviour and concentration inequalities. 


\subsection{Expectation estimates}

We will start with some estimates of the expected operator norm. In the proof of our results we use an argument introduced by Meckes, but apply it conditionally.

Following [12, let us first note that $T_{n}$ is a submatrix of the infinite Laurent matrix

$$
L_{n}=\left[X_{i-1} \mathbf{1}_{\{|i-j| \leq n-1\}}\right]_{i, j \in \mathbb{Z}},
$$

so $\left\|T_{n}\right\| \leq\left\|L_{n}\right\|$, where by $\left\|L_{n}\right\|$ we denote the operator norm of $L_{n}$ acting in the standard way on $\ell_{2}(\mathbb{Z})$. If we use the Fourier basis to identify $\ell_{2}(\mathbb{Z})$ with $L_{2}[0,1]$, it turns out that $L_{n}$ corresponds to a multiplication operator, with the multiplier

$$
f(t)=X_{0}+2 \sum_{j=1}^{n-1} \cos (2 \pi j t) X_{j}
$$

and thus $\left\|L_{n}\right\|=\|f\|_{\infty}$.

Theorem 4. If $X_{0}, X_{1}, \ldots$ are independent mean zero random variables, then

$$
\mathbb{E}\left\|T_{n}\right\| \leq \mathbb{E}\left\|L_{n}\right\| \leq C \sqrt{\sum_{i=0}^{n-1} \mathbb{E} X_{i}^{2}} \sqrt{\log n},
$$

where $C$ is an absolute constant.

Proof. By the arguments preceding the formulation of the theorem and the standard symmetrization inequality, we have

$$
\mathbb{E}\left\|T_{n}\right\| \leq \mathbb{E}\left\|L_{n}\right\| \leq 2 \mathbb{E} \sup _{t \in[0,1]}\left|Y_{t}\right|
$$

where

$$
Y_{t}=X_{0} \varepsilon_{0}+2 \sum_{j=1}^{n-1} \cos (2 \pi j t) \varepsilon_{j} X_{j}
$$

and $\varepsilon_{1}, \varepsilon_{2}, \ldots$ is a sequence of i.i.d. Rademacher variables, independent of $X_{j}$ 's. Using the fact that Rademacher variables are subgaussian and Dudley's entropy bound, conditionally on $X_{i}$ 's, one gets

$$
\mathbb{E}_{\varepsilon} \sup _{t \in[0,1]}\left|Y_{t}\right| \leq\left|Y_{0}\right|+C \int_{0}^{\infty} \sqrt{\log N([0,1], d, s)} d s
$$

where $C$ is an absolute constant,

$$
d\left(t_{1}, t_{2}\right)=\left\|Y_{t_{1}}-Y_{t_{2}}\right\|_{2}=2 \sqrt{\sum_{j=1}^{n-1} X_{j}^{2}\left[\cos \left(2 \pi j t_{1}\right)-\cos \left(2 \pi j t_{2}\right)\right]^{2}}
$$

and $N([0,1], d, s)$ is the smallest cardinality of an $s$-net in $[0,1]$ with the metric $d$ (see e.g. [14], chapter 2.2). 
We have $\operatorname{diam}([0,1], d) \leq 4 \sqrt{\sum_{j=1}^{n-1} X_{j}^{2}}=: D$. Moreover

$$
d\left(t_{1}, t_{2}\right) \leq 4 \pi \sqrt{\sum_{j=1}^{n-1} j^{2} X_{j}^{2}}\left|t_{1}-t_{2}\right|,
$$

so

$$
N([0,1], d, s) \leq C \frac{A}{s},
$$

for $s \leq D$, where $A=\sqrt{\sum_{j=1}^{n-1} X_{j}^{2} j^{2}}$.

Therefore

$$
\begin{aligned}
\int_{0}^{\infty} & \sqrt{\log N([0,1], d, s)} d s \leq \int_{0}^{D} \sqrt{\log \frac{C A}{s}} d s \\
& =\frac{C A}{\sqrt{2}} \int_{\sqrt{2 \log (C A / D)}}^{\infty} t^{2} e^{-t^{2} / 2} d t \\
& \leq \frac{C A}{\sqrt{2}}(\sqrt{2 \log (C A / D)}+\sqrt{2 \pi}) e^{-\log (C A / D)} \\
& \leq D \sqrt{\log (C A / D)}+\sqrt{\pi} D .
\end{aligned}
$$

We have $A / D \leq \sqrt{\sum_{j=1}^{n-1} j^{2}} \leq C n^{3 / 2}$, so the above inequality together with (8) implies

$$
\mathbb{E}_{\varepsilon} \sup _{t \in[0,1]}\left|Y_{t}\right| \leq\left|Y_{0}\right|+C \sqrt{\sum_{j=1}^{n-1} X_{j}^{2}} \sqrt{\log n} .
$$

Taking into account that $Y_{0}=X_{0} \varepsilon_{0}+2 \sum_{j=1}^{n-1} X_{j} \varepsilon_{j}$, integrating with respect to the variables $X_{j}$ 's and using Jensen's inequality, we get

$$
\mathbb{E}\left\|L_{n}\right\| \leq C \sqrt{\sum_{j=0}^{n-1} \mathbb{E} X_{j}^{2}} \sqrt{\log n} .
$$

The following lemma is a partial converse to the above theorem. It also relies on arguments from [12].

Lemma 1. If $X_{0}, X_{1}, \ldots$, are i.i.d. and

$$
\limsup _{n \rightarrow \infty} \frac{\mathbb{E}\left\|T_{n}\right\|}{\sqrt{n \log n}}<\infty,
$$

then $\mathbb{E} X_{i}^{2}<\infty$. 
Proof. Without loss of generality we can assume that $X_{i}$ 's are symmetric with positive variance. Thus for $R$ large enough $X_{j} \mathbf{1}_{\left\{\left|X_{j}\right| \leq R\right\}}$ is not identically zero.

Let $\varepsilon_{1}, \varepsilon_{2}, \ldots$ be i.i.d. Rademacher variables, independent of $X_{i}$ 's. By assumption, for some $C<\infty$,

$$
\mathbb{E}\left\|T_{n}\right\| \leq C \sqrt{n \log n} .
$$

By another argument from [12] (see the proof of Theorem 3 therein), we have

$$
\left\|T_{n}\right\| \geq \sup _{t \in[0,1]}\left|X_{0}+\sum_{j=1}^{n-1} 2(1-j / n) X_{j} \cos (2 \pi j t)\right| .
$$

Thus, using symmetry of $X_{j}$ 's and the contraction principle, we obtain that for each $R<\infty$,

$$
\mathbb{E}\left\|T_{n}\right\| \geq \mathbb{E}_{X} \mathbb{E}_{\varepsilon} \sup _{t \in[0,1]}\left|\sum_{j=0}^{\lfloor n / 2\rfloor} \varepsilon_{j} X_{j} \mathbf{1}_{\left\{\left|X_{j}\right| \leq R\right\}} \cos (2 \pi j t)\right| .
$$

Now, by Proposition 6 of [12, we obtain for $R$ large enough

$$
\mathbb{E}\left\|T_{n}\right\| \geq c \mathbb{E}\left\|\left(a_{j}\right)_{j=1, \ldots,\lfloor n / 2\rfloor}\right\|_{2} \sqrt{\log \frac{\left\|\left(a_{j}\right)_{j=1, \ldots,\lfloor n / 2\rfloor}\right\|_{2}}{\left\|\left(a_{j}\right)_{j=1, \ldots,\lfloor n / 2\rfloor}\right\|_{4}}},
$$

where $a_{j}=X_{j} \mathbf{1}_{\left\{\left|X_{j}\right| \leq R\right\}}$. Let $A=\mathbb{E} X_{0}^{2} \mathbf{1}_{\left\{\left|X_{0}\right| \leq R\right\}}$. By the law of large numbers, for $n$ large enough (depending on $R$ ), the random variable on the right hand side is with probability at least $1 / 2$ greater than or equal to

$$
\sqrt{n} \sqrt{A / 2} \sqrt{\log \frac{n^{1 / 2} \sqrt{A / 2}}{n^{1 / 4} R}} \geq \sqrt{n} \sqrt{A / 2} \sqrt{\log \left(n^{1 / 8} \sqrt{A / 2}\right)} .
$$

Thus, for $n$ large enough (depending on $R$ and $A$ ), we have

$$
\sqrt{n A} \sqrt{\log n^{1 / 16}} \leq \sqrt{n A} \sqrt{\log \left(n^{1 / 8} \sqrt{A}\right)} \leq \sqrt{2} C c^{-1} \sqrt{n \log n},
$$

which gives $A \leq 32 C^{2} c^{-2}$. Since $R$ is arbitrary, it implies that $\mathbb{E} X_{0}^{2} \leq 32 C^{2} c^{-2}$.

\subsection{Almost sure behaviour}

Before we proceed to the analysis of the almost sure behaviour of the operator norm of $T_{n}$, let us introduce some notation that will be used throughout the rest of the paper.

For every positive integer $n$ and $i=0, \ldots, n-1$, let $A_{i}^{(n)}=\left[\mathbf{1}_{\{|j-l|=i\}}\right]_{j, l \leq n}$. We thus have

$$
T_{n}=\sum_{i=0}^{n-1} X_{i} A_{i}^{(n)}
$$

This basic relation will allow us to apply to $T_{n}$ the results for sums of independent random variables, presented in Section 2 . 


\subsubsection{The non i.i.d. case}

As the first example of application of Theorem 2 we will prove the following

Proposition 1. If $X_{1}, X_{2}, \ldots$ are independent (not necessarily i.i.d.), $\mathbb{E} X_{n}=0$ and for some $\alpha>1$, $\sup _{n} \mathbb{E}\left|X_{n}\right|^{2}\left[\log \log \left(\left|X_{n}\right| \vee e^{e}\right)\right]^{\alpha}<\infty$, then with probability 1 ,

$$
\limsup _{n \rightarrow \infty} \frac{\left\|T_{n}\right\|}{\sqrt{n \log n}}=\limsup _{n \rightarrow \infty} \frac{\mathbb{E}\left\|T_{n}\right\|}{\sqrt{n \log n}}<\infty
$$

Proof. The inequality follows immediately from Theorem 4 We will first prove, that

$$
\limsup _{n \rightarrow \infty} \frac{\left\|T_{n}\right\|}{\sqrt{n \log n}} \leq \limsup _{n \rightarrow \infty} \frac{\mathbb{E}\left\|T_{n}\right\|}{\sqrt{n \log n}} \text { a.s. }
$$

It turns out that to obtain the inverse of (10), we will need a corresponding result for Laurent matrices, i.e.

$$
\limsup _{n \rightarrow \infty} \frac{\left\|L_{n}\right\|}{\sqrt{n \log n}} \leq \limsup _{n \rightarrow \infty} \frac{\mathbb{E}\left\|L_{n}\right\|}{\sqrt{n \log n}} \text { a.s. }
$$

The proofs of both statements are very similar. Therefore we will present in detail only the argument for $T_{n}$ and then we will indicate all the changes one has to make in order to obtain (11).

Let us denote $A=\lim \sup \mathbb{E}\left\|T_{n}\right\| / \sqrt{n \log n}$. To obtain (10), it is enough to show that for all $\varepsilon>0$,

$$
\lim _{n \rightarrow \infty} \mathbb{P}\left(\sup _{k>n}\left\|T_{k}\right\| / \sqrt{k \log k}>A+\varepsilon\right)=0
$$

We have for $\theta>1$,

$$
\begin{aligned}
\mathbb{P}\left(\sup _{k>n}\left\|T_{k}\right\| / \sqrt{k \log k}>A+\varepsilon\right) & \\
\leq & \sum_{k \geq\lfloor\log n / \log \theta\rfloor} \mathbb{P}\left(\max _{\theta^{k} \leq i<\theta^{k+1}}\left\|T_{i}\right\|>(A+\varepsilon) \sqrt{\theta^{k} \log \theta^{k}}\right) \\
& =\sum_{k \geq\lfloor\log n / \log \theta\rfloor} \mathbb{P}\left(\left\|T_{\theta^{k+1}}\right\|>(A+\varepsilon) \sqrt{\theta^{k} \log \theta^{k}}\right),
\end{aligned}
$$

where in the last inequality we used the fact that $\left\|T_{i}\right\|$ is increasing with $i$ (since $T_{i}$ is a submatrix of $T_{i+1}$ ). Now, for $\eta$ small enough and $k$ large enough, we have

$$
\begin{aligned}
(A+\varepsilon / 2) \sqrt{\theta^{k} \log \theta^{k}} & \geq(A+\varepsilon / 4)(1+2 \eta) \sqrt{\theta^{k} \log \theta^{k}} \\
& \geq(1+2 \eta) \mathbb{E}\left\|T_{\theta^{k+1}}\right\| \sqrt{\theta^{-1} \frac{k \log \theta}{(k+1) \log \theta}},
\end{aligned}
$$


which for $\theta$ close to one and $k$ large enough is greater than or equal to $(1+$ $\eta) \mathbb{E}\left\|T_{\theta^{k+1}}\right\|$. Thus, to prove (12), it is enough to show that

$$
\sum_{k=1}^{\infty} \mathbb{P}\left(\left\|T_{\theta^{k+1}}\right\| \geq \mathbb{E}\left\|T_{\theta^{k+1}}\right\|(1+\eta)+\varepsilon \sqrt{\theta^{k} \log \theta^{k}} / 2\right)<\infty
$$

for arbitrary $\theta>1$ and $\varepsilon, \eta>0$. Let us now denote $\Psi(x)=x[\log \log (x \vee M)]^{\alpha}$ and $\Phi(x)=x /[\log \log (x \vee M)]^{\alpha}$, where $M$ is a parameter and note that for $M$ large enough, $\Psi$ is convex and nondecreasing and $\Phi$ is nondecreasing. Moreover for some constant $K$ (depending on $M$ and $\alpha$ ) and all $x \geq 0$,

$$
K+\Phi(\Psi(x)) \geq x / K
$$

We will now take into account the relation (9) to treat $T_{n}$ as a sum of independent variables in the space of matrices and apply Theorem 2, Let us notice that since $\left\|A_{i}^{(n)}\right\| \leq 2, \sigma^{2}$ from this theorem is bounded by $4 \theta^{k+1} \sup _{i<\theta^{k+1}} \mathbb{E} X_{i}^{2} \leq$ $K_{1} \theta^{k+1}$ for some constant $K_{1}$ (depending on the supremum in the assumption of the proposition). Therefore, applying Theorem 2 with $p=2$, we obtain

$$
\begin{aligned}
\mathbb{P}\left(\left\|T_{\theta^{k+1}}\right\|\right. & \left.\geq \mathbb{E}\left\|T_{\theta^{k+1}}\right\|(1+\eta)+\varepsilon \sqrt{\theta^{k} \log \theta^{k}} / 2\right) \\
& \leq e^{-\frac{k \varepsilon^{2} \log \theta}{K_{2}}}+K_{2} \frac{\mathbb{E} \max _{i<\theta^{k+1}\left|X_{i}\right|^{2}}}{\varepsilon^{2} \theta^{k} k \log \theta} .
\end{aligned}
$$

The series corresponding to the first term on the right hand side is clearly convergent. As for the second term, by the integrability assumption on $X_{i}$ 's, we have for some constants $C_{1}, C_{2}$,

$$
\begin{aligned}
K^{-1} \mathbb{E} \max _{i<\theta^{k+1}}\left|X_{i}\right|^{2} & \leq K+\Phi\left(\Psi\left(\mathbb{E} \max _{i<\theta^{k+1}}\left|X_{i}\right|^{2}\right)\right) \leq K+\Phi\left(\mathbb{E} \sum_{i<\theta^{k+1}} \Psi\left(\left|X_{i}\right|^{2}\right)\right) \\
& \leq K+\Phi\left(\theta^{k+1} C_{1}\right) \leq K+\frac{\theta^{k+1} C_{2}}{\log ^{\alpha} k},
\end{aligned}
$$

which shows that also the other series is convergent, as

$$
\sum_{k=1}^{\infty} \frac{1}{k \log ^{\alpha} k}<\infty
$$

for $\alpha>1$. This proves (12) and thus also (10).

Let us now indicate the changes one has to make in the above argument, to prove (11). First, let us notice that the matrices $L_{n}$ are sums of independent variables with values in $\mathcal{B}\left(\ell_{2}(\mathbb{Z})\right)$ - the space of all bounded operators on the Hilbert space $\ell_{2}(\mathbb{Z})$. Indeed, we have

$$
L_{n}=\sum_{i=0}^{n-1} X_{i} A_{i}
$$


where $A_{i}=\left[\mathbf{1}_{\{|i-j|=i\}}\right]_{i, j \in \mathbb{Z}}$.

The space $\mathcal{B}\left(\ell_{2}(\mathbb{Z})\right)$ is not separable, but one can still apply Theorem 1 since the norm on this space is expressible as a supremum over a countable set of functionals, so one can approximate it by norms in separable spaces. The second difference is the lack of monotonicity of $\left\|L_{n}\right\|$ with respect to $n$, which prevents us from using a counterpart of the equality (13). This is however not a real problem, since, denoting $A=\limsup \left\|L_{n}\right\| / \sqrt{n \log n}$, one can write

$$
\begin{aligned}
\mathbb{P}\left(\sup _{k>n}\left\|L_{k}\right\| / \sqrt{k \log k}>A+\varepsilon\right) & \\
\leq & \sum_{k \geq\lfloor\log n / \log \theta\rfloor} \mathbb{P}\left(\max _{\theta^{k} \leq i<\theta^{k+1}}\left\|L_{i}\right\|>(A+\varepsilon) \sqrt{\theta^{k} \log \theta^{k}}\right) \\
& \leq \sum_{k \geq\lfloor\log n / \log \theta\rfloor} \mathbb{P}\left(\max _{i \leq \theta^{k+1}}\left\|L_{i}\right\|>(A+\varepsilon) \sqrt{\theta^{k} \log \theta^{k}}\right)
\end{aligned}
$$

and use the full strength of Theorem 1, which gives a bound for the tail of maxima of partial sums. The rest of the proof is the same as for $T_{n}$ (note that we still have $\left.\left\|A_{i}\right\| \leq 2\right)$.

What remains to be proved is the inverse inequality, i.e.

$$
\limsup _{n \rightarrow \infty} \frac{\left\|T_{n}\right\|}{\sqrt{n \log n}} \geq \limsup _{n \rightarrow \infty} \frac{\mathbb{E}\left\|T_{n}\right\|}{\sqrt{n \log n}} .
$$

This can be established again by arguments similar as in [6], Section 4.3 (see also [1, Theorem 7). First, let us define $X_{n}^{\prime}=X_{n} \mathbf{1}_{\left\{\left|X_{n}\right| \leq \sqrt{n \log n}\right\}}$ and let $T_{n}^{\prime}$ (resp. $L_{n}^{\prime}$ ) be the Toeplitz (resp. Laurent) matrix generated by $X_{n}^{\prime}$. We have

$$
\limsup _{n \rightarrow \infty} \frac{\left\|T_{n}\right\|}{\sqrt{n \log n}}=\limsup _{n \rightarrow \infty} \frac{\left\|T_{n}^{\prime}\right\|}{\sqrt{n \log n}} \text { a.s. }
$$

and

$$
\limsup _{n \rightarrow \infty} \frac{\mathbb{E}\left\|T_{n}\right\|}{\sqrt{n \log n}}=\limsup _{n \rightarrow \infty} \frac{\mathbb{E}\left\|T_{n}^{\prime}\right\|}{\sqrt{n \log n}} .
$$

To prove the first equality, it is enough to notice that by the Chebyshev inequality

$$
\begin{aligned}
\sum_{n} \mathbb{P}\left(X_{n} \neq X_{n}^{\prime}\right) & \leq \sum_{n} \mathbb{P}\left(\left|X_{n}\right|>\sqrt{n \log n}\right) \\
& \leq \sum_{n} C \frac{\mathbb{E}\left|X_{n}\right|^{2}\left[\log \log \left(\left|X_{n}\right| \vee e^{e}\right)\right]^{\alpha}}{n[\log n][\log \log n]^{\alpha}}<\infty
\end{aligned}
$$

which by the Borel-Cantelli Lemma implies that with probability $1, X_{n}=X_{n}^{\prime}$ for large $n$. The equality (16) follows by (9), an analogous formula for $T_{n}^{\prime}$ and the fact that $\left\|A_{i}^{(n)}\right\| \leq 2$. 
Let us now turn to the proof of (17). Notice that

$$
\begin{aligned}
\left|\mathbb{E}\left\|T_{n}\right\|-\mathbb{E}\left\|T_{n}^{\prime}\right\|\right| & \leq \sum_{i=0}^{n-1}\left\|A_{i}^{(n)}\right\| \mathbb{E}\left|X_{i}\right| \mathbf{1}_{\left\{\left|X_{i}\right|>\sqrt{i \log i}\right\}} \\
& \leq 2 \sum_{i=0}^{n} \mathbb{E}\left|X_{i}\right| \mathbf{1}_{\left\{\left|X_{i}\right|>\sqrt{i \log i}\right\}} \leq K \sum_{i=0}^{n-1} \frac{\mathbb{E}\left|X_{i}\right|^{2}}{\sqrt{i \log i}} \\
& =o(\sqrt{n \log n}),
\end{aligned}
$$

which already implies (17).

To prove (15), it is thus sufficient to show that $\sup _{n}\left\|L_{n}^{\prime}\right\| / \sqrt{n \log n}$ is integrable, since then, by Fatou's lemma (recall that $\left\|T_{n}^{\prime}\right\| \leq\left\|L_{n}^{\prime}\right\|$ ), we get

$$
\mathbb{E} \limsup _{n} \frac{\left\|T_{n}^{\prime}\right\|}{\sqrt{n \log n}} \geq \underset{n}{\limsup } \frac{\mathbb{E}\left\|T_{n}^{\prime}\right\|}{\sqrt{n \log n}}
$$

which allows us to finish the proof, since by (10), (16) and (17), we have

$$
\limsup _{n} \frac{\left\|T_{n}^{\prime}\right\|}{\sqrt{n \log n}} \leq \limsup _{n} \frac{\mathbb{E}\left\|T_{n}^{\prime}\right\|}{\sqrt{n \log n}} \text { a.s. }
$$

To prove the desired integrability, notice that by Theorem 4] (11), (14), the inequality $\left\|A_{i}\right\| \leq 2$ and the fact that with probability $1, X_{n}=X_{n}^{\prime}$ for large $n$, we have

$$
\sup _{n} \frac{\left\|L_{n}^{\prime}\right\|}{\sqrt{n \log n}}<\infty \text { a.s. }
$$

Moreover $\mathbb{E} \sup _{n}\left\|A_{n} X_{n}^{\prime}\right\| / \sqrt{n \log n} \leq 2$. By Proposition 6.12. from [10], this implies that also

$$
\mathbb{E} \sup _{n} \frac{\left\|L_{n}^{\prime}\right\|}{\sqrt{n \log n}}<\infty
$$

which ends the proof.

We would also like to remark that if one considers higher iterates of logarithm, the assumptions of Proposition 11 may be weakened, for instance to $\sup _{n} \mathbb{E}\left|X_{n}\right|^{2}\left[\log \log \left(\left|X_{n}\right| \vee M\right)\right]\left[\log \log \log \left(\left|X_{n}\right| \vee M\right)\right]^{\alpha}<\infty$ for some $\alpha>1$ (the proof is essentially the same, the only difference is in the involved series). The proposition is however no longer true if we assume only $\sup _{n} \mathbb{E}\left|X_{n}\right|^{2}\left[\log \log \left(\left|X_{n}\right| \mathrm{V}\right.\right.$ $e)]<\infty$. To see this, notice that since $\left\|T_{n}\right\| \geq\left|X_{n-1}\right|$, by the Borel-Cantelli Lemma a necessary condition for the almost sure finiteness of the limsup in question is

$$
\sum_{n} \mathbb{P}\left(\left|X_{n}\right| \geq C \sqrt{n \log n}\right)<\infty
$$

for some $C<\infty$, which is not true for instance if $X_{n}= \pm \sqrt{n[\log n][\log \log \log n]}$ with probability $p_{n}=(n[\log n][\log \log n][\log \log \log n])^{-1}$ and 0 with probability $1-2 p_{n}$. 


\subsubsection{The i.i.d. case. Proof of main results.}

We will now prove the main results of the article, announced in the Introduction. We state them again below as Theorem 5 and Corollary 3 . The proofs go along the same lines as in the non i.i.d. case. The main tool is Theorem 1 (now we can actually use the original formulation from [6], with sums of $p$-th moments instead of the $p$-th moment of the maximum of $\left|X_{i}\right|$ 's).

Theorem 5. Assume that $X_{0}, X_{1}, X_{2}, \ldots$ are i.i.d. random variables. If $\mathbb{E} X_{0}=$ 0 and $0<\mathbb{E} X_{0}^{2}<\infty$, then

$$
\lim _{n \rightarrow \infty} \frac{\left\|T_{n}\right\|}{\mathbb{E}\left\|T_{n}\right\|}=1 \text { a.s. }
$$

Proof. Just as the proof of Proposition 1 the argument is influenced by the work of Einmahl and Li. The first step is the truncation.

Let us define $X_{n}^{\prime}=X_{n} \mathbf{1}_{\left\{\left|X_{n}\right| \leq \sqrt{n \log n\}}\right.}$. We have

$$
\begin{aligned}
\sum_{n} \mathbb{P}\left(X_{n} \neq X_{n}^{\prime}\right) & =\sum_{n} \mathbb{P}\left(X_{0}>\sqrt{n \log n}\right) \\
& =\mathbb{E} \sum_{n} \mathbf{1}_{\left\{n \log n<X_{0}^{2}\right\}} \leq K \mathbb{E} X_{0}^{2}<\infty
\end{aligned}
$$

so with probability $1, X_{n}=X_{n}^{\prime}$ for large $n$.

By Theorem 4, we have $\mathbb{E}\left\|T_{n}\right\| \leq C \sqrt{n \log n}$ for some $C<\infty$ (depending on the distribution of $X_{0}$ ). Also, by Theorem 3 of [12], symmetrization inequalities and Jensen's inequality, we have

$$
\begin{aligned}
\mathbb{E}\left\|T_{n}\right\| & \geq 2^{-1}\left\|\sum_{i=0}^{n-1} A_{i}^{(n)} X_{i} \varepsilon_{i}\right\| \geq 2^{-1} \mathbb{E}\left|X_{0}\right|\left\|\sum_{i=0}^{n-1} A_{i}^{(n)} \varepsilon_{i}\right\| \\
& \geq \tilde{c} \mathbb{E}\left|X_{0}\right| \sqrt{n \log n}=c \sqrt{n \log n},
\end{aligned}
$$

where $\tilde{c}$ is universal and $c=\tilde{c} \mathbb{E}\left|X_{0}\right|$.

Let $T_{n}^{\prime}$ be the Toeplitz matrix corresponding to $X_{0}^{\prime}, \ldots, X_{n-1}^{\prime}$. Since $X_{n}=$ $X_{n}^{\prime}$ for large $n$ and by the above estimates $\mathbb{E}\left\|T_{n}\right\| \rightarrow \infty$, it is enough to prove that with probability 1 ,

$$
\lim _{n} \frac{\left\|T_{n}^{\prime}\right\|}{\mathbb{E}\left\|T_{n}\right\|}=1 .
$$


We also have

$$
\begin{aligned}
\left|\mathbb{E}\left\|T_{n}\right\|-\mathbb{E}\left\|T_{n}^{\prime}\right\|\right| & \leq \mathbb{E}\left|\left\|T_{n}\right\|-\left\|T_{n}^{\prime}\right\|\right| \leq \mathbb{E}\left\|T_{n}-T_{n}^{\prime}\right\| \\
& =\mathbb{E}\left\|\sum_{i=0}^{n-1} A_{i}^{(n)} X_{i} \mathbf{1}_{\left\{\left|X_{i}\right|>\sqrt{i \log i}\right\}}\right\| \\
& \leq 2 \mathbb{E}\left|X_{0}\right|+2 \sum_{i=1}^{n-1} \mathbb{E}\left|X_{i}\right| \mathbf{1}_{\left\{\left|X_{i}\right|>\sqrt{i \log i}\right\}} \\
& \leq 4 \mathbb{E}\left|X_{0}\right|+2 \mathbb{E}\left|X_{0}\right|^{2} \sum_{i=2}^{n-1} \frac{1}{\sqrt{i \log i}}=o(\sqrt{n \log n})=o\left(\mathbb{E}\left\|T_{n}\right\|\right),
\end{aligned}
$$

which shows that $\mathbb{E}\left\|T_{n}\right\| / \mathbb{E}\left\|T_{n}^{\prime}\right\| \rightarrow 1$, so it is enough to prove that with probability 1 ,

$$
\lim _{n} \frac{\left\|T_{n}^{\prime}\right\|}{\mathbb{E}\left\|T_{n}^{\prime}\right\|}=1 .
$$

For simplicity let us denote $s_{n}=\mathbb{E}\left\|T_{n}^{\prime}\right\|$. Notice that by the above inequalities, we have

$$
c \sqrt{n \log n} \leq s_{n} \leq C \sqrt{n \log n}
$$

for some $0<c<C<\infty$. Fix $\theta>1$ (but close to 1 ) and let $m_{n}$ be the smallest integer $k$ such that $s_{k}>\theta^{n}$. We have $m_{n} \rightarrow \infty$.

Notice that we have

$$
s_{n+1}-s_{n} \leq \mathbb{E} \sqrt{\sum_{i=0}^{n} 2\left(X_{i}^{\prime}\right)^{2}} \leq \sqrt{2 n \mathbb{E} X_{0}^{2}}=o\left(s_{n}\right) .
$$

Thus

$$
1<\frac{s_{m_{n}}}{\theta^{n}} \leq \frac{s_{m_{n}}}{s_{m_{n}-1}} \leq 1+\alpha_{n},
$$

where $\alpha_{n} \rightarrow 0$, so $s_{m_{n}} / \theta^{n} \rightarrow 1$. In particular $s_{m_{n+1}} / s_{m_{n}} \rightarrow \theta$.

Having established these preliminary facts, we can proceed with the proof of (19). We will show separately that $\lim \sup \left\|T_{n}^{\prime}\right\| / s_{n} \leq 1$ and $\lim \inf \left\|T_{n}^{\prime}\right\| / s_{n} \geq 1$ a.s.

We want to prove that for every $\varepsilon>0$,

$$
\lim _{n \rightarrow \infty} \mathbb{P}\left(\sup _{k>n} \frac{\left\|T_{k}^{\prime}\right\|}{s_{k}}>1+\varepsilon\right)=0
$$

and

$$
\lim _{n \rightarrow \infty} \mathbb{P}\left(\inf _{k>n} \frac{\left\|T_{k}^{\prime}\right\|}{s_{k}}<1-\varepsilon\right)=0,
$$


which will follow if for some $\theta>1$ we show that

$$
\sum_{k} \mathbb{P}\left(\max _{m_{k} \leq i<m_{k+1}} \frac{\left\|T_{i}^{\prime}\right\|}{s_{i}}>1+\varepsilon\right)<\infty
$$

and

$$
\sum_{k} \mathbb{P}\left(\min _{m_{k} \leq i<m_{k+1}} \frac{\left\|T_{i}^{\prime}\right\|}{s_{i}}<1-\varepsilon\right)<\infty
$$

Similarly as in the proof of Proposition 1 we can now take advantage of the monotonicity of $\left\|T_{n}^{\prime}\right\|$ (and $s_{n}$ ), which allows us to replace the above conditions by

$$
\sum_{k} \mathbb{P}\left(\left\|T_{m_{k+1}}^{\prime}\right\|>(1+\varepsilon) s_{m_{k}}\right)<\infty
$$

and

$$
\sum_{k} \mathbb{P}\left(\left\|T_{m_{k}}^{\prime}\right\|<(1-\varepsilon) s_{m_{k+1}}\right)<\infty
$$

Let us now notice that since $\mathbb{E} X_{i}=0$, we have

$$
\begin{aligned}
\left\|\mathbb{E} T_{n}^{\prime}\right\| & =\left\|\sum_{i=0}^{n-1} A_{i}^{(n)} \mathbb{E} X_{i} \mathbf{1}_{\left\{\left|X_{i}\right| \leq \sqrt{i \log i\}}\right.}\right\|=\left\|\sum_{i=0}^{n-1} A_{i}^{(n)} \mathbb{E} X_{i} \mathbf{1}_{\left\{\left|X_{i}\right|>\sqrt{i \log i\}}\right.}\right\| \\
& \leq \mathbb{E}\left\|\sum_{i=0}^{n-1} A_{i}^{(n)} X_{i} \mathbf{1}_{\left\{\left|X_{i}\right|>\sqrt{i \log i\}}\right.}\right\|=o\left(\mathbb{E}\left\|T_{n}\right\|\right)=o\left(s_{n}\right),
\end{aligned}
$$

where in the third equality we used (18).

Since $s_{m_{k+1}} / s_{m_{k}} \rightarrow \theta$, for large $k$,

$$
\begin{aligned}
\left\|T_{m_{k}}^{\prime}\right\| & \geq\left\|T_{m_{k}}^{\prime}-\mathbb{E} T_{m_{k}}^{\prime}\right\|-\varepsilon s_{m_{k+1}} / 4, \\
\left\|T_{m_{k+1}}^{\prime}\right\| & \leq\left\|T_{m_{k+1}}^{\prime}-\mathbb{E} T_{m_{k+1}}^{\prime}\right\|+\varepsilon s_{m_{k}} / 4,
\end{aligned}
$$

so (21) and (22) will follow if we show that

$$
\sum_{k} \mathbb{P}\left(\left\|T_{m_{k+1}}^{\prime}-\mathbb{E} T_{m_{k+1}}^{\prime}\right\|>(1+3 \varepsilon / 4) s_{m_{k}}\right)<\infty
$$

and

$$
\sum_{k} \mathbb{P}\left(\left\|T_{m_{k}}^{\prime}-\mathbb{E} T_{m_{k}}^{\prime}\right\|<(1-3 \varepsilon / 4) s_{m_{k+1}}\right)<\infty .
$$

Finally for $\theta \simeq 1$ (depending on $\varepsilon$ ) and large $k$ we can write

$$
\begin{aligned}
\mathbb{E}\left\|T_{m_{k+1}}^{\prime}-\mathbb{E} T_{m_{k+1}}^{\prime}\right\| & \leq s_{m_{k+1}}(1+\varepsilon / 4) \leq s_{m_{k}}(1+\varepsilon / 2), \\
\mathbb{E}\left\|T_{m_{k}}^{\prime}-\mathbb{E} T_{m_{k}}^{\prime}\right\| & \geq s_{m_{k}}(1-\varepsilon / 4) \geq s_{m_{k+1}}(1-\varepsilon / 2),
\end{aligned}
$$


so the proof of (23) and (24) can be reduced to showing that

$$
\sum_{k} \mathbb{P}\left(\left\|T_{m_{k+1}}^{\prime}-\mathbb{E} T_{m_{k+1}}^{\prime}\right\|>\left(1+\eta_{1}\right) \mathbb{E}\left\|T_{m_{k+1}}^{\prime}-\mathbb{E} T_{m_{k+1}}^{\prime}\right\|+\varepsilon s_{m_{k}} / 8\right)<\infty
$$

and

$$
\sum_{k} \mathbb{P}\left(\left\|T_{m_{k}}^{\prime}-\mathbb{E} T_{m_{k}}^{\prime}\right\|<\left(1-\eta_{2}\right) \mathbb{E}\left\|T_{m_{k}}^{\prime}-\mathbb{E} T_{m_{k}}^{\prime}\right\|-\varepsilon s_{m_{k+1}} / 8\right)<\infty
$$

where

$$
\begin{aligned}
& 1+\eta_{1}=(1+5 \varepsilon / 8)(1+\varepsilon / 2)^{-1}, \\
& 1-\eta_{2}=(1-5 \varepsilon / 8)(1-\varepsilon / 2)^{-1} .
\end{aligned}
$$

Now we can use Theorem 2 with $p=3$ to obtain

$$
\begin{aligned}
& \mathbb{P}\left(\left\|T_{m_{k+1}}^{\prime}-\mathbb{E} T_{m_{k+1}}^{\prime}\right\|>\left(1+\eta_{1}\right) \mathbb{E}\left\|T_{m_{k+1}}^{\prime}-\mathbb{E} T_{m_{k+1}}^{\prime}\right\|+\varepsilon s_{m_{k}} / 8\right) \\
& \leq \exp \left(-\frac{\varepsilon^{2} s_{m_{k}}^{2}}{K m_{k+1}}\right)+K \frac{m_{k+1} \mathbb{E}\left|X_{0}\right|^{3} \mathbf{1}_{\left\{\left|X_{0}\right| \leq \sqrt{m_{k+1} \log m_{k+1}}\right\}}}{s_{m_{k}}^{3} \varepsilon^{3}}
\end{aligned}
$$

Similarly

$$
\begin{aligned}
& \mathbb{P}\left(\left\|T_{m_{k}}^{\prime}-\mathbb{E} T_{m_{k}}^{\prime}\right\|<\left(1-\eta_{2}\right)\left\|T_{m_{k}}^{\prime}-\mathbb{E} T_{m_{k}}^{\prime}\right\|-\varepsilon s_{m_{k+1}} / 8\right) \\
& \leq K \exp \left(-\frac{\varepsilon^{2} s_{m_{k+1}}^{2}}{K m_{k}}\right)+K \frac{m_{k} \mathbb{E}\left|X_{0}\right|^{3} \mathbf{1}_{\left\{\left|X_{0}\right| \leq \sqrt{m_{k} \log m_{k}}\right\}}}{s_{m_{k+1}}^{3} \varepsilon^{3}} .
\end{aligned}
$$

By (20) and the definition of $s_{k}$ we now obtain, that for large $k, \sqrt{m_{k} \log m_{k}} \simeq$ $\theta^{k}$ and $s_{m_{k}} \simeq \theta^{k}$, so we also have $m_{k} \simeq \theta^{2 k} / k$ (where by $\simeq$ we mean that the quotient of the left and right hand side is bounded away from 0 and infinity by some constants, perhaps depending on $\theta$, but independent of $k$ ). Thus the right hand sides of (25) and (26) are bounded by

$$
\begin{aligned}
& K \exp \left(-\frac{\varepsilon^{2} \theta^{2 k}}{K \theta^{2 k+2} k^{-1}}\right)+K \frac{\theta^{2 k+2} \mathbb{E}\left|X_{0}\right|^{3} \mathbf{1}_{\left\{\left|X_{0}\right| \leq C \theta^{k}\right\}}}{\theta^{3 k} k \varepsilon^{3}} \\
& \leq K \exp \left(-\frac{\varepsilon^{2} k}{K \theta^{2}}\right)+K \frac{\theta^{2} \mathbb{E}\left|X_{0}\right|^{3} \mathbf{1}_{\left\{\left|X_{0}\right| \leq C \theta^{k}\right\}}}{\theta^{k} k \varepsilon^{3}} .
\end{aligned}
$$

The series corresponding to the first term on the right hand side is clearly convergent. As for the other term, we have

$$
\begin{aligned}
\sum_{k} \frac{\mathbb{E}\left|X_{0}\right|^{3} \mathbf{1}_{\left\{\left|X_{0}\right| \leq C \theta^{k}\right\}}}{\theta^{k} k} & \leq \mathbb{E}\left|X_{0}\right|^{3} \sum_{k: \theta^{k} \geq\left|X_{0}\right| / C} \theta^{-k} \leq \mathbb{E}\left|X_{0}\right|^{3} \cdot \frac{K C}{\left|X_{0}\right|} \\
& =K C \mathbb{E}\left|X_{0}\right|^{2}<\infty
\end{aligned}
$$

which shows (21) and (22) and thus proves the theorem. 
Remark Actually, in the above proof one may as well use the inequality for bounded variables (e.g. Corollary 1). It is enough to notice that all the other calculations will still work if we truncate the variables $X_{i}$ at the level $\sqrt{n}$ instead of $\sqrt{n \log n}$.

As a corollary we obtain a law of large numbers for the i.i.d. case, proven originally in [4].

Corollary 2. Assume that $X_{i}$ are i.i.d. random variables, such that $\mathbb{E} X_{0}^{2}<\infty$ and $\mathbb{E} X_{0}=m$. Then

$$
\lim _{n \rightarrow \infty} \frac{\left\|T_{n}\right\|}{n}=|m|
$$

Proof. The argument we present comes from [12]. We repeat it for the sake of completeness. Let $T_{n}^{\prime}$ be the matrix generated by the variables $X_{i}-m$, $i=0, \ldots, n-1$. By Theorems 4 and 5 , we have

$$
\limsup _{n \rightarrow \infty} \frac{\left\|T_{n}^{\prime}\right\|}{\sqrt{n \log n}}<\infty .
$$

But $T_{n}^{\prime}=T_{n}-m I_{n}$, where $I_{n}$ is the $n \times n$ matrix of ones. Since $\left\|I_{n}\right\|=n$, we have

$$
\frac{\left|\left\|T_{n}\right\|-\right| m|n|}{n} \leq \frac{\left\|T_{n}^{\prime}\right\|}{n} \rightarrow 0,
$$

which proves the corollary.

Remark A similar corollary for Toeplitz matrices generated by independent but not identically distributed random variables with the same mean may be derived from Proposition 1. Also, if we assume for instance that $\mathbb{E}\left|X_{n}\right|$ is bounded away from 0 , we can combine the proofs of Proposition 1 and Theorem 5 to obtain the law of large numbers (with the normalization $\mathbb{E}\left\|T_{n}\right\|$ ) in the centered, non i.i.d. case.

Combining Theorem [5 with Lemma 1 gives the necessary and sufficient conditions for the $\lim \sup \left\|T_{n}\right\| / \sqrt{n \log n}$ to be almost surely finite.

Corollary 3. Assume that $X_{i}$ are i.i.d. random variables. Then

$$
\limsup _{n \rightarrow \infty} \frac{\left\|T_{n}\right\|}{\sqrt{n \log n}}<\infty \text { a.s. }
$$

if and only if $\mathbb{E} X_{0}=0$ and $\mathbb{E} X_{0}^{2}<\infty$.

Proof. The first part follows from Theorems 4 and 5 . To prove the second part it is enough to show that $\mathbb{E} X_{0}^{2}<\infty$, since then Corollary 2 gives $\mathbb{E} X_{0}=0$. To prove the finiteness of the second moment, without loss of generality we may 
assume that the variable is symmetric. The square integrability will follow by Lemma 1 if we prove that

$$
\limsup _{n \rightarrow \infty} \frac{\mathbb{E}\left\|T_{n}\right\|}{\sqrt{n \log n}}<\infty .
$$

This can be proven in the same way as part (a) of Lemma 4.1 in 6] (this lemma deals with partial sums of i.i.d. variables, whereas in our case $T_{n}=$ $\sum_{i=0}^{n-1} A_{i}^{(n)} X_{i}$, however the fact that $\left\|A_{i}^{(n)}\right\|$ are bounded, uniformly in $i$ and $n$, makes the same argument valid also in our situation).

Problem In view of results for other types of random matrices, a natural question arises, whether $\frac{\left\|T_{n}\right\|}{\sqrt{n \log n}}$ is almost surely convergent. From Theorem 5 it follows that this is equivalent to the existence of $\lim \frac{\mathbb{E}\left\|T_{n}\right\|}{\sqrt{n \log n}}$.

\subsection{Some concentration inequalities}

We would like to conclude by presenting some concentration of measure results for $\left\|T_{n}\right\|$.

Again, we will look at the matrix $T_{n}$ as a sum of independent random variables in a Banach space. However, since the weak variance $\sigma^{2}$, defined in Section 2 is (in the i.i.d. case) up to a universal constant the same as the so called strong variance, defined in our situation as

$$
\Sigma^{2}=\sum_{i=0}^{n-1} \mathbb{E}\left\|A_{i}\right\|^{2} X_{i}^{2} \leq 4 \sum_{i=1}^{n} \mathbb{E} X_{i}^{2}
$$

we will pass to sums of real random variables. At the cost of increasing some constants by a universal factor (in the i.i.d. case), this will allow us to obtain concentration around $\mathbb{E}\left\|T_{n}\right\|$ rather than bounds on the probability of deviation from $(1 \pm \eta) \mathbb{E}\left\|T_{n}\right\|$.

Our main technical tool will be the following symmetrization lemma, proved in 22] (it actually seems to be a part of the folklore, but we haven't been able to find any references).

Lemma 2. Let $\varphi: \mathbb{R} \rightarrow \mathbb{R}$ be a convex function and $G=f\left(Y_{1}, \ldots, Y_{n}\right)$, where $Y_{1}, \ldots, Y_{n}$ are independent random variables with values in a measurable space $\mathcal{E}$. Denote

$$
G_{i}=f\left(Y_{1}, \ldots, Y_{i-1}, \tilde{Y}_{i}, Y_{i+1}, \ldots, Y_{n}\right),
$$

where $\left(\tilde{Y}_{1}, \ldots, \tilde{Y}_{n}\right)$ is an independent copy of $\left(Y_{1}, \ldots, Y_{n}\right)$ and assume that

$$
\left|G-G_{i}\right| \leq F_{i}\left(Y_{i}, \tilde{Y}_{i}\right)
$$

for some functions $F_{i}: \mathcal{E}^{2} \rightarrow \mathbb{R}, i=1, \ldots, n$. Then

$$
\mathbb{E} \varphi(G-\mathbb{E} G) \leq \mathbb{E} \varphi\left(\sum_{i=1}^{n} \varepsilon_{i} F_{i}\left(Y_{i}, \tilde{Y}_{i}\right)\right),
$$


where $\varepsilon_{1}, \ldots, \varepsilon_{n}$ is a sequence of independent Rademacher variables, independent of $\left(Y_{i}\right)_{i=1}^{n}$ and $\left(\tilde{Y}_{i}\right)_{i=1}^{n}$.

Thus, whenever we have a concentration inequality for sums of centered real variables, which has been obtained by (or can be rephrased as) an estimate for expectations of one or many convex functions (like the Lagrange transform or moment estimates), by using the above Lemma we can obtain a corresponding result for more general variables. In most cases the inequalities for sums of Banach space valued variables obtained with this method will be much worse than the optimal ones, since they lose the information about the weak variance, in our case however the weak variance satisfies

$$
\begin{aligned}
\sigma^{2} & =\sup _{\|\alpha\|_{2},\|\beta\|_{2} \leq 1} \sum_{k=0}^{n-1}\left(\sum_{i, j:|i-j|=k} \alpha_{i} \beta_{j}\right)^{2} \mathbb{E} X_{k}^{2} \\
& =\sup _{\|\alpha\|_{2},\|\beta\|_{2},\left\|\gamma_{2}\right\|_{2} \leq 1}\left(\sum_{k} \sum_{i, j:|i-j|=k} \alpha_{i} \beta_{j} \gamma_{k}\left(\mathbb{E} X_{k}^{2}\right)^{1 / 2}\right)^{2} \\
& =\sup _{\|\gamma\|_{2} \leq 1}\left\|\left[\gamma_{|i-j|}\left(\mathbb{E} X_{|i-j|}^{2}\right)^{1 / 2}\right]_{i, j \leq n}\right\|^{2}
\end{aligned}
$$

and in the i.i.d. case the choice of $\gamma_{k}=1 / \sqrt{n}$ for all $k$ gives $\sigma^{2} \geq n \mathbb{E} X_{0}^{2}$.

In the following proposition we present inequalities for Toeplitz matrices generated by various classes of independent variables. The proof relies on translating proper inequalities for real variables into a moment form (which in this case can be easily achieved with integration by parts), using Lemma 2 and then going back to tail estimates by optimizing in $p$ the Chebyshev inequality for $p$-th moments. We will skip the details which are quite standard but lengthy. The real line inequalities we use are the $\psi_{2}$ inequality for centered variables and Theorem 3 .

Proposition 2. Let $X_{0}, \ldots, X_{n-1}$ be independent random variables and let $T_{n}$ the corresponding random Toeplitz matrix. Then

(a) if $\left\|X_{i}\right\|_{\psi_{2}}<\infty$ for all $i$, then for all $t \geq 0$,

$$
\mathbb{P}\left(\left|\left\|T_{n}\right\|-\mathbb{E}\left\|T_{n}\right\|\right| \geq t\right) \leq K \exp \left(-\frac{t^{2}}{K \sum_{i=0}^{n-1}\left\|X_{i}\right\|_{\psi_{2}}^{2}}\right),
$$

where $K$ is a universal constant,

(b) if for some $\alpha \in(0,1],\left\|X_{i}\right\|_{\psi_{\alpha}}<\infty$ for all $i$, then

$$
\mathbb{P}\left(\left|\left\|T_{n}\right\|-\mathbb{E}\left\|T_{n}\right\|\right| \geq t\right) \leq 2 \exp \left[-\frac{1}{K_{\alpha}} \min \left(\frac{t^{2}}{\Sigma^{2}}, \frac{t}{\left\|\max _{i}\left|X_{i}\right|\right\|_{\psi_{\alpha}}}\right)\right],
$$

where $\Sigma^{2}$ is defined by formula (27) and $K_{\alpha}$ depends only on $\alpha$. 
Remark Obviously one can apply the same scheme to other inequalities. We would also like to remark that in the non i.i.d. case one may prefer to use Theorem 3 for sums of independent Banach space valued variables. At the moment we do not know whether also in this case $\sigma^{2}$ and $\Sigma^{2}$ are of the same order.

\subsection{Final remarks}

In the article we restrict our attention to symmetric Toeplitz matrices. However, the same methods provide analogous results for nonsymmetric Toeplitz matrices $\left[X_{i-j}\right]_{i \leq i, j \leq n}$ where $X_{i}, i \in \mathbb{Z}$ are independent random variables. Therefore, the methods apply also to random Hankel matrices $\left[X_{j+k-1}\right]_{1 \leq j, k \leq n}$, since (as noticed in 5], Remark 1.2.), the singular values of $H_{n}$ are the same as of the nonsymmetric Toeplitz matrix, obtained from $H_{n}$ by reversing the order of the rows. The results about the expected operator norm of the nonsymmetric Toeplitz matrix translate thus directly. As for Theorem 5 one has to be a little bit more careful. This result does not translate formally, since the Toeplitz matrices corresponding to $H_{n}$ are generated by a permutation of the original sequence of random variables. One can however still use all the concentration of measure results and simply repeat the proof.

\section{References}

[1] de Acosta A., Kuelbs J., Ledoux, M. An inequality for the law of the iterated logarithm. Probability in Banach spaces, IV (Oberwolfach, 1982), 1-29, Lecture Notes in Math., 990, Springer, Berlin, 1983. MR0707506.

[2] Adamczak R. A tail inequality for suprema of unbounded empirical processes with applications to Markov chains. Submitted.

[3] Bai Z.D. Methodologies in spectral analysis of large-dimensional random matrices, a review. Statist. Sinica, 9 (3), 611-677, 1999. MR1711663

[4] Bose A., Sen A. Spectral norm of random large dimensional noncentral Toeplitz and Hankel matrices. Electron. Comm. Probab., 12, 29-35, 2007. MR2284045

[5] Bryc W., Dembo A., Jiang T., Spectral measure of large Hankel, Markov and Toeplitz matrices. Ann. Probab., 34 (1), 1-38, 2006. MR2206341

[6] Einmahl U., Li D., Characterization of LIL behavior in Banach space. To appear in Trans. Amer. Math. Soc.

[7] Fuk, D. H., Nagaev, S. V. Probabilistic inequalities for sums of independent random variables. Teor. Verojatnost. i Primenen. 16, 660-675, 1971. MR0293695 
[8] Giné E., Latała R., Zinn J., Exponential and moment inequalities for $U$ statistics. In High Dimensional Probability II, 13-38. Progr. Probab. 47. Birkhauser, Boston, MA, 2000. MR1857312.

[9] Hammond C., Miller S.J., Distribution of eigenvalues for the ensemble of real symmetric Toeplitz matrices. J. Theoret. Probab., 18, 537-566, 2005. MR2167641.

[10] Ledoux M., Talagrand M., Probability in Banach Spaces. Isoperimetry and processes., Volume 23 of Ergebnisse der Mathematik und ihrer Grenzgebiete (3). Springer-Verlag, Berlin 1991. MR1102015

[11] Klein T., Rio E., Concentration around the mean for maxima of empirical processes. Ann. Probab. 33, 1060-1077, 2005. MR2135312

[12] Meckes M., On the spectral norm of a random Toeplitz matrix, Elect. Comm. in Probab., 12, 315-325, 2007. MR2342710

[13] Talagrand M. New concentration inequalities in product spaces. Invent. Math. 126, 1996, no. 3, 505-563. MR1419006.

[14] van der VaArt, Aad W., Wellner, Jon A. Weak convergence and empirical processes. With applications to statistics. Springer Series in Statistics. Springer-Verlag, New York, 1996. MR1385671 